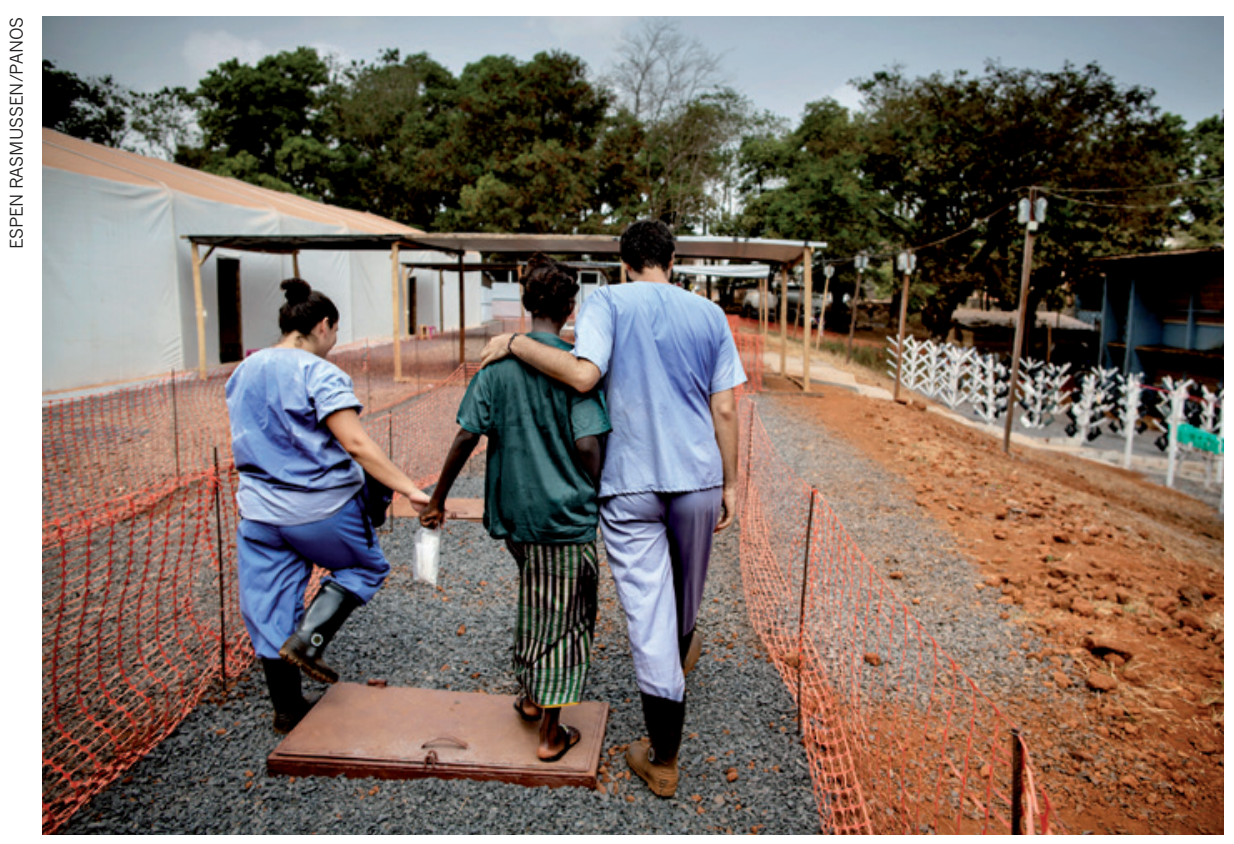

An Ebola survivor is helped out of a clinic in Sierra Leone built by Médecins Sans Frontières.

PUBLIC HEALTH

\title{
Ebola failures prompt WHO rethink
}

\section{Health agency's annual meeting will address shortcomings in outbreak response highlighted by West Africa crisis.}

\section{BY ERIKA CHECK HAYDEN}

$\mathrm{A}$ $s$ West Africa's Ebola epidemic wanes, a fever is building for reform of the World Health Organization (WHO). When the 194 member states of the United Nations body meet on 18-26 May in Geneva, Switzerland, for the 68th World Health Assembly, they will consider a number of proposals intended to make the WHO more nimble and effective at responding to fast-moving public-health crises.

At stake is the organization's future as the primary responder to global health emergencies. In September 2014, after the WHO's slow response in West Africa, the UN created a separate organization, the UN Mission for Ebola Emergency Response, to tackle the outbreak. And last month, UN secretary-general Ban Ki-moon appointed a panel on managing future health crises that is expected to issue a report by the end of this year. Observers note that previous WHO failures led to the formation of independent initiatives such as GAVI, the Vaccine Alliance, which vaccinates children in the developing world, and the Global Fund to Fight AIDS, Tuberculosis and Malaria.

"The worry would be that some other group will take contingency planning and emergency response away from WHO," says infectiousdisease specialist Barry Bloom of the Harvard T. H. Chan School of Public Health in Boston, Massachusetts. "That has been the trend and leads back to the fundamental question of what is the role of WHO."

WHO director-general Margaret Chan has acknowledged that the agency made big mistakes in the Ebola epidemic. The outbreak revealed "inadequacies and shortcomings in this organization's administrative, managerial and technical infrastructures," she said in a January speech, which asked for reforms to the WHO and for an external review of the agency's performance. A draft of the review released on 8 May concluded that "WHO does not have the operational capacity or culture to deliver a full emergency public health response."

The main reforms up for discussion in Geneva include creating a US\$100-million fund for response to fast-moving events such as the Ebola epidemic; setting up an international cadre of first responders to outbreaks; and setting guidelines for how aid groups, foundations, academic institutions and corporations can take part in WHO meetings. Such guidelines have been in the works for years as global public-health spending has increasingly shifted to non-governmental organizations. But the importance of private entities was brought to the fore by the Ebola outbreak, with groups such as Médecins Sans Frontières (also known as Doctors Without Borders) proving to be more effective than the WHO or governments.

The agency is also asking member states to boost its budget by $8 \%$ for $2016-17$, after having received flat funding since 2012 . And Chan wants to strengthen the International Health Regulations - rules agreed by member states in 2005 that require countries to set up basic outbreak-response mechanisms - but there are no specific proposals for how this would occur.

Many reformers attending the meeting are worried that even the enormity of the Ebola epidemic will not motivate the WHO membership to adopt reform, and are pessimistic about the effectiveness of such changes if they are approved. Some of the same ideas were proposed - but never adopted - after outbreaks of the respiratory syndrome SARS, H5N1 'bird flu' and H1N1 'swine flu' in the 2000s.

"I want to emphasize the importance of making these changes now, while the epidemic is fresh in our mind, and not wait, because the political momentum is with us now, and it will fade the same way it did with SARS and H1N1," says Lawrence Gostin, a health-law and policy specialist at Georgetown University in Washington DC who serves on a WHO committee charged with reforming the organization.

That sentiment is shared by Adam KamradtScott, a health-security specialist at the University of Sydney in Australia. But he is pessimistic about the probability of change and frustrated about poor resources. "I doubt that we will see any significant reforms emerge," he says. "While governments complain on the one hand that the organization isn't effective, they also don't give the WHO sufficient resources or authority to do the job they've asked it to do."

The WHO relies on voluntary contributions for more than $70 \%$ of its budget; it is unlikely that countries would provide for the new task force or contingency fund at needed levels, says Kamradt-Scott.

Yet in the wake of the Ebola outbreak, governments and international organizations have signalled a willingness to provide funding to strengthen preparedness for health crises, says Josh Michaud, associate director of global health policy at the non-profit Kaiser Family Foundation in Washington DC. He notes that the United States last year approved more than $\$ 5$ billion in emergency funding for Ebola response and that the World Bank has pledged to provide $\$ 650$ million to help countries to rebuild after the epidemic. The United Kingdom has also said that it would provide $\$ 10$ million to the WHO's proposed contingency fund.

"It's not easy or cheap to build up these capacities," Michaud says. "But there are signs that Ebola has sparked real change." - 